\title{
Reduced glucose-induced first-phase insulin release is a danger signal that predicts diabetes
}

\author{
Gordon C. Weir and Susan Bonner-Weir \\ Section on Islet Cell and Regenerative Biology, Joslin Diabetes Center, Harvard Medical School, Boston Massachusetts, USA.
}

\begin{abstract}
During progression to both types 1 and 2 diabetes (T1D, T2D), there is a striking loss of glucose-induced first-phase insulin release (FPIR), which is known to predict the onset of T1D. The contribution of reduced $\beta$ cell mass to the onset of hyperglycemia remains unclear. In this issue of the $\mathrm{JCl}$, Mezza et al. report on their study of patients with pancreatic neoplasms before and after partial pancreatectomy to evaluate the impact of reduced $\beta$ cell mass on the development of diabetes. The authors found that reduced FPIR predicted diabetes when $50 \%$ of the pancreas was removed. These findings suggest that low or absent FPIR indicates that $\beta$ cell mass can no longer compensate for increased insulin needs. Notably, clinicians may use reduction of FPIR as a warning that progression to T2D is underway.
\end{abstract}

\section{The earliest stages of diabetes} progression

The loss of glucose-induced first-phase insulin release (FPIR) is a striking finding seen in the earliest stages of progression to diabetes (1-3). This FPIR loss has been thoroughly studied in individuals at risk for developing autoimmune type 1 diabetes (T1D), identified via MHC haplotypes associated with susceptibility to diabetes and antibodies against $\beta$ cell antigens (4). It has become clear that FPIR loss predicts that full-blown T1D is probably only months away. Now, thanks to a study by Mezza et al. in this issue of the JCI (5), we find that reduction of FPIR can also predict the appearance of diabetes after surgically removing $50 \%$ of the pancreas for neoplasms. This finding strongly suggests that a reduction of FPIR has similar predictive value for those at risk for T2D.

We know that diabetes develops when pancreatic $\beta$ cells cannot produce enough insulin to maintain normal blood glucose levels despite varying degrees of insulin sensitivity. This failure of $\beta$ cells to compensate is caused by the combined effects of insufficient $\beta$ cell mass and dysfunctional insulin secretion. To evaluate these variables, Mezza et al. studied a group of 33 subjects without impaired glucose tolerance (IGT) or diabetes who underwent $50 \%$ partial pancreatectomy for neoplasm (5). Key well-understood studies were performed before and after surgery, including oral glucose tolerance tests (OGTTs) and hyperglycemic clamps (HCs), followed by arginine stimulation. Previous studies have shown that removing $50 \%$ of the pancreas of living donors to provide islets for transplantation increased the risk of progressing to diabetes (6). However, there has been no way to predict which patients would progress. In the Mezza et al. study, after surgery, 11 of the initial 33 remained normoglycemic and 13 had IGT. The nine who went on to develop diabetes had striking reductions of FPIR before surgery. Insulin sensitivity, as determined by plasma insulin levels and the Masuda

\section{Related Article: https://doi.org/10.1172/JCl146788}

Conflict of interest: GCW has served on the scientific advisory board (SAB) of Semma Therapeutics and received stocks and serves on the SAB for BetaO2 Ltd.

Copyright: () 2021, American Society for Clinical Investigation.

Reference information: J Clin Invest. 2021;131(12):e150022. https://doi.org/10.1172/JCI150022.

index, was measured and changed little after the surgery (5).

\section{Understanding functional $\beta$ cell mass and $\beta$ cell reserve}

Studies on autopsied pancreases show that $\beta$ cell mass is about $40 \%$ to $60 \%$ lower in those from patients with T2D than in controls of comparable weight $(7,8)$. While it is often assumed that this lower $\beta$ cell mass results from increases in $\beta$ cell death, the underlying problem for some could be deficient $\beta$ cell generation earlier in life. The concept of functional $\beta$ cell mass is important. We know that $\beta$ cells in the presence of hyperglycemia have profound impairments of insulin secretion that are accompanied by major changes in gene expression (9, 10). Some call this dedifferentiation, but it might be more accurately described as an altered phenotype or disruption of $\beta$ cell identity. This change in phenotype appears to be caused by a combination of increased work by $\beta$ cells as they struggle to compensate and the effects of the hyperglycemia resulting from an inadequate $\beta$ cell mass. The concept of $\beta$ cell reserve can be viewed as the capacity of $\beta$ cells to compensate for increased demand when being pushed to secrete more insulin (9). This compensation is accomplished by more $\beta$ cells becoming active, enhanced secretion from cells already active, and possibly by some compensatory growth, mainly from self-replication. The critical point is that $\beta$ cell reserve is highly protective while it lasts, but it can be used up, leaving no backup capacity to cope with further loss of $\beta$ cells or increases in insulin demand. At that threshold, $\beta$ cell mass becomes inadequate.

\section{Mechanistic changes as $\beta$ cell function deteriorates}

Because glucose has such dominant effects on $\beta$ cells, it is reasonable to assume that glucose, working through an efficient feedback mechanism, drives $\beta$ cell compensation for insulin resistance. An example of glucose feedback on $\beta$ cell 

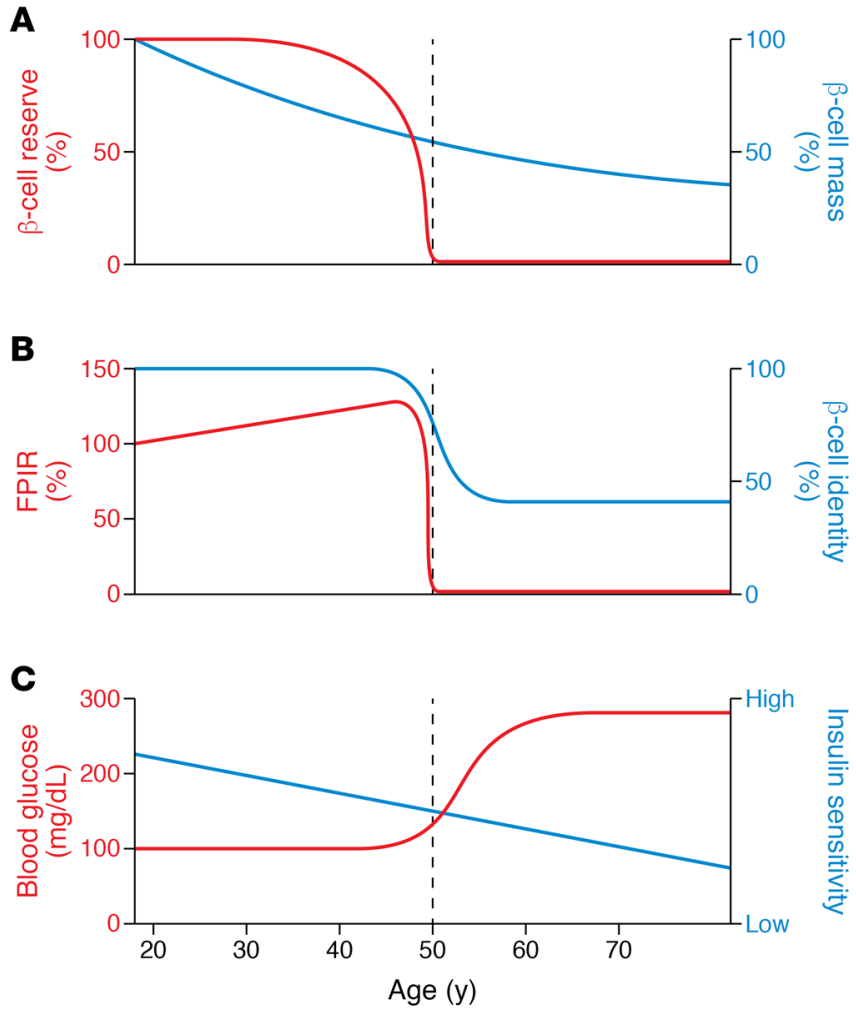

Figure 1. A hypothetical path to $\beta$ cell failure in T2D. In this example, a hypothetical individual develops T2D at age 50, showing the loss of glucose-induced FPIR (B) as a marker of impending $\beta$ cell failure. $\beta$ Cell failure occurs after years of successful compensation that kept blood glucose levels normal in the face of climbing insulin resistance (shown as falling insulin sensitivity) (C) and gradually declining $\beta$ cell mass (A). Note the early increase in FPIR, which contributes to the compensation for insulin resistance. With time, $\beta$ cell reserve is expended, leaving $\beta$ cell mass (A) as inadequate, resulting in the onset of $\beta$ cell failure, shown as a marked fall in FPIR associated with changes in $\beta$ cell gene expression. Glucose levels (C) then rise and cause further disruption of $\beta$ cell identity and function. There can be variations of this general pattern; for example, the ratios of insulin sensitivity to cell mass can be very different, but lead to the same point of decompensation marked by loss of FPIR. Even without insulin resistance, some can go through this progression as their $\beta$ cells fail

growth is shown in two studies showing that mice with glucokinase haploinsufficiency failed to increase $\beta$ cell replication, which is normally associated with high-fat diets $(11,12)$. These findings indicate that changes in glucose metabolism can influence gene expression, which supports the concept that changes in glucose flux, with or without hyperglycemia, can lead to the changes in $\beta$ cell phenotype that are responsible for the marked functional deterioration. They also support the conclusion that glucose exerts toxic effects at least in part through changes in gene expression, further justifying use of the term glucose toxicity (13).

In spite of the logic behind these assumptions, we have a limited understanding of where the trouble responsible for the loss of FPIR lies. Insulin secretion can be driven by a $\mathrm{K}_{\mathrm{ATP}}$-dependent mech- anism, also known as triggering, and by a $\mathrm{K}_{\mathrm{ATP}}$-independent mechanism, known as amplification $(14,15)$. Defective $\mathrm{K}_{\mathrm{ATP}}$ channel signaling should be considered a prime suspect for impaired insulin secretion. Since increases in the ATP/ADP ratio drive the channel to close, impaired ATP delivery to the $\mathrm{K}_{\mathrm{ATP}}$ channel could explain the loss of FPIR. Production of ATP in the cytoplasm via glycolysis and in the mitochondria through oxidative phosphorylation and/or through anapleurosis largely determines $\mathrm{K}_{\mathrm{ATP}}$ activity.

\section{Differences in FPIR loss between T1D and T2D}

The loss of FPIR in T1D has been well described in longitudinal observational studies of individuals with known risk because they possess susceptible MHC haplotype and antibodies to $\beta$ cell anti- gens (4). Clinicians often note the loss of FPIR when glucose levels are in the normal range or only slightly elevated. Once the reduction of FPIR is found, full-blown diabetes can be expected shortly, suggesting an acceleration of autoimmune killing. This conclusion is supported by the finding of increased circulating unmethylated DNA from the insulin gene, which is thought to result from $\beta$ cell death (16), and further supported by emerging data showing increased expression of both class I and II MHC in $\beta$ cells as their phenotype changes, which could make the $\beta$ cells more susceptible to autoimmunity $(17,18)$. Thus, when $\beta$ cell mass is inadequate and glucose levels start to climb, changes in the $\beta$ cell phenotype will lead to both impaired insulin secretion and increased vulnerability.

The findings by Mezza et al. (5) show that, as has been shown for progression to T1D, a reduction in FPIR can predict progression to diabetes after partial pancreatectomy, which has implications for the progression to T2D. The deterioration of $\beta$ cell secretion starts when $\beta$ cell mass is inadequate and $\beta$ cell reserve has been expended and can lead to diabetes. However, we do not yet have evidence that the rate of cell death is accelerated by these changes in phenotype. Although data are limited, it appears from autopsy studies that $\beta$ cell mass declines in established T2D about $1.5 \%$ per year, a rather modest rate.(8). The changes in gene expression cover a variety of pro- and antiapoptotic factors, but the increased expression of the prosurvival Hifla pathway $(18,19)$ may help explain how progression to T2D is considerably slower than that for T1D.

These findings shine light on the importance of low or absent FPIR, which serves as a dramatic announcement that $\beta$ cell mass can no longer compensate and that the elegant secretory machinery is faltering and on the verge of collapse (Figure 1). We can now raise questions about the implications of this danger signal for prevention strategies.

\section{Acknowledgments}

Funding for this study was provided by JDRF (2-SRA-2018-527-S-B to GCW), NIH (R01 DK110390 to SBW), the NIH-funded Joslin Diabetes Research Center (PO DK036836), and the Diabetes Research and Wellness Foundation. 
Address correspondence to: Gordon C. Weir, Section on Islet Cell and Regenerative Biology, Joslin Diabetes Center, One Joslin Place, Boston, Massachusetts 02215, USA. Phone: 1.617.732.2581; Email: gordon.weir@joslin.harvard.edu.

1. Brunzell JD, et al. Relationships between fasting plasma glucose levels and insulin secretion during intravenous glucose tolerance tests. JClin Endocrinol Metab. 1976;42(2):222-229.

2. Vardi $P$, et al. Predictive value of intravenous glucose tolerance test insulin secretion less than or greater than the first percentile in islet cell antibody positive relatives of type 1 (insulin-dependent) diabetic patients. Diabetologia. 1991;34(2):93-102.

3. Cerasi E, et al. Decreased sensitivity of the pancreatic beta cells to glucose in pre-diabetic and diabetic subjects. A glucose dose-response study. Diabetes. 1971;21(4):224-234.

4. Sosenko JM, et al. Acceleration of the loss of the first-phase insulin response during the progression to type 1 diabetes in diabetes prevention trial-type 1 participants. Diabetes. 2013;62(12):4179-4183.
5. Mezza, et al. Pancreaticoduodenectomy model demonstrates a fundamental role of dysfunctional $\beta$ cells in predicting diabetes. J Clin Invest. 2021;131(12):e146788.

6. Seaquist ER, Robertson RP. Effects of hemipancreatectomy on pancreatic alpha and beta cell function in healthy human donors. JClin Invest. 1992;89(6):1761-1766.

7. Butler AE, et al. Beta-cell deficit and increased beta-cell apoptosis in humans with type 2 diabetes. Diabetes. 2003;52(1):102-110.

8. Rahier J, et al. Pancreatic beta-cell mass in European subjects with type 2 diabetes. Diabetes Obes Metab. 2008;10(Suppl 4):32-42.

9. Weir GC, et al. Inadequate $\beta$-cell mass is essential for the pathogenesis of type 2 diabetes. Lancet Diabetes Endocrinol. 2020;8(3):249-256.

10. Weir GC, Bonner-Weir S. Islet $\beta$ cell mass in diabetes and how it relates to function, birth, and death. Ann N Y Acad Sci. 2013;1281:92-105.

11. Terauchi Y, et al. Glucokinase and IRS-2 are required for compensatory beta cell hyperplasia in response to high-fat diet-induced insulin resistance. J Clin Invest. 2007;117(1):246-257.

12. Porat $S$, et al. Control of pancreatic $\beta$ cell regeneration by glucose metabolism. Cell Metab.
2011;13(4):440-449.

13. Weir GC. Glucolipotoxicity, $\beta$-cells, and diabetes: the emperor has no clothes. Diabetes. 2020;69(3):273-278.

14. Lewandowski SL, et al. Pyruvate kinase controls signal strength in the insulin secretory pathway. Cell Metab. 2020;32(5):736-750.

15. Henquin JC. Regulation of insulin secretion: a matter of phase control and amplitude modulation. Diabetologia. 2009;52(5):739-751.

16. Herold KC, et al. $\beta$ cell death and dysfunction during type 1 diabetes development in at-risk individuals. J Clin Invest. 2015;125(3):1163-1173.

17. Russell MA, et al. HLA Class II antigen processing and presentation pathway components demonstrated by transcriptome and protein analyses of Islet $\beta$-cells from donors with type 1 diabetes. Diabetes. 2019;68(5):988-1001.

18. Ebrahimi AG, et al. Beta cell identity changes with mild hyperglycemia: Implications for function, growth, and vulnerability. Mol Metab. 2020;35:100959.

19. Montemurro C, et al. IAPP toxicity activates HIF1 $\alpha$ /PFKFB3 signaling delaying $\beta$-cell loss at the expense of $\beta$-cell function. Nat Commun. 2019;10(1):2679. 